\title{
Short communication: Retinoic acid plus prolactin to synergistically increase specific casein gene expression in MAC-T cells
}

\author{
H. Y. Lee, ${ }^{*}$ Y. T. Heo, ${ }^{\star}$ S. E. Lee, ${ }^{*}$ K. C. Hwang, ${ }^{*}$ H. G. Lee,† S. H. Choi, ${ }^{\star} \ddagger$ and N. H. Kim ${ }^{* 1}$ \\ *Department of Animal Science, Chungbuk National University, Chungbuk 361-763, Republic of Korea \\ †Department of Animal Science, Konkuk University, Seoul 143-701, Republic of Korea \\ ‡Department of Animal Science, Texas A\&M University, College Station 77843
}

\section{ABSTRACT}

Mammary alveolar (MAC-T) cells, an established bovine mammary epithelial cell line, are frequently used to investigate differentiation. A lactogenic phenotype in these cells is induced by treatment with a combination of hydrocortisone, insulin, and prolactin (PRL). The effect of the vitamin A derivative retinoic acid (RA), which induces differentiation in many cells, has not been studied in MAC-T cells. The objective of this study was to evaluate the differentiation potential of RA $(1 \mu M)$ in MAC-T cells and to examine the effect of combined treatment with RA $(1 \mu M)$ and PRL $(5 \mu \mathrm{g} /$ $\mathrm{mL}$ ). Although RA treatment alone inhibited MAC-T cell proliferation, co-treatment of RA with PRL increased cell growth compared with the control group (treated with $1 \mu \mathrm{g} / \mathrm{mL}$ hydrocortisone and $5 \mu \mathrm{g} / \mathrm{mL}$ insulin). The ratio of $B c l$ to $B a x$ mRNA was decreased in the RA treatment compared with RA+PRL or control. Retinoic acid-induced differentiation of MAC-T cells was associated with an increase in the mRNA expres-

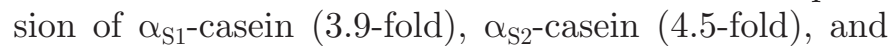
$\beta$-casein (4.4-fold) compared with the control group. Expression of $\alpha_{S_{1}}$-casein, $\alpha_{S 2}$-casein, and $\beta$-casein was increased 12.9-fold, 11.9-fold, and 19.3-fold, respectively, following treatment with RA and PRL combined compared with the control group. These results demonstrate that RA induces differentiation of MAC-T cells and acts synergistically with PRL to increase specific casein gene expression.

Key words: MAC-T cell line, retinoic acid, differentiation, casein gene expression

\section{Short Communication}

Bovine mammary epithelial cell lines, such as mammary alveolar (MAC-T) cells, have been used extensively to study apoptosis and autophagy in the immune response, lipid metabolism, and mammalian lactation

Received July 15, 2012.

Accepted February 26, 2013.

${ }^{1}$ Corresponding author: nhkim@chungbuk.ac.kr
(Peterson et al., 2004; Al-Bataineh et al., 2010; Zhao et al., 2010; Sobolewska et al., 2011). In the field of animal biotechnology, these cell lines represent a convenient validation system for evaluating foreign gene expression and the production of human targeted proteins luteinizing hormone (Pajot-Augy et al., 1997) and telomerase reverse transcriptase (He et al., 2011) in milk. Bovine mammary epithelial cells have been used as a model system for studying lactation and testing mammary tissue-specific expression vectors (Huynh et al., 1991; Zavizion et al., 1996), as well as for the production of bioactive substances and target proteins with the mammary tissue-specific casein promoter (Brophy et al., 2003).

Bovine MAC-T cells partially differentiate into $\beta$-casein-secreting cells when treated with prolactin (PRL), a peptide hormone that is primarily synthesized in lactotrophic cells in the anterior pituitary of vertebrates (Lyons, 1958). However, Lyons (1958) suggested that the efficiency of differentiation induction of MAC-T cells into $\beta$-casein-secreting cells by PRL is only approximately $30 \%$. Therefore, increasing the efficiency of MAC-T cell differentiation may offer a more feasible system for the validation of targeted protein production by the $\beta$-casein promoter.

Retinoic acid (RA) is a lipid-soluble signaling molecule derived from vitamin A (retinol) that regulates a diverse array of biological processes, including cellular proliferation, differentiation, and apoptosis throughout embryonic development (Kumar and Duester, 2011). Retinoic acid also plays an important role in the regulation of immune responses (McCullough et al., 1999). Hundreds of genes are known to be regulated by RA via complex processes regulated on multiple levels, and RA is currently used clinically for the treatment of several diseases (Fukasawa et al., 2012; Pecori et al., 2012). Mehta and Moon (1985) suggested that the biological response may depend on the ability of the target cells to both metabolize the retinoid and express the minimal concentration of cellular retinoic acid binding protein for binding to the active metabolite. Therefore, the hypothesis addressed in this study was that supplementation with RA would improve the efficiency of 
differentiation of MAC-T cells. To test this hypothesis, we investigated the effects of RA treatment alone and with PRL on the expression of $\alpha_{\mathrm{S}_{1}}$-casein, $\alpha_{\mathrm{S}_{2}}$-casein, and $\beta$-casein mRNA as markers of the differentiation potential of MAC-T cells. To our knowledge, this is the first report demonstrating a synergistic increase in specific casein gene expression induced by the treatment of MAC-T cells with the combination of PRL and RA. These findings may contribute to the development of an advanced validation system for targeted protein expression in mammalian cells.

The MAC-T cells were grown in Dulbecco's modified Eagle's medium (DMEM; Hyclone, Waltham, MA) containing $10 \%$ fetal bovine serum (FBS; Hyclone), $100 \mathrm{IU} / \mathrm{mL}$ penicillin, and $100 \mu \mathrm{g} / \mathrm{mL}$ streptomycin (Sigma, St. Louis, MO) at $37^{\circ} \mathrm{C}$ in an atmosphere of $5 \% \mathrm{CO}_{2}$ and air. For differentiation, MAC-T cells were cultured in serum-free DMEM for $16 \mathrm{~h}$ and then treated with 5\% FBS as a basal medium. Control treatment included the addition of $5 \mu \mathrm{g} / \mathrm{mL}$ insulin (Sigma) and $1 \mu \mathrm{g} / \mathrm{mL}$ hydrocortisone (Sigma) to the basal medium. Treated cells were cultured in the control medium plus $5 \mu \mathrm{g} / \mathrm{mL}$ PRL (Sigma) or $1 \mu M \mathrm{RA}$ (Sigma), or both, in DMEM containing 5\% FBS. Medium was changed daily and cells were harvested at d 1, 2, 4, and 8 .

The MAC-T cells were cultured to $80 \%$ confluency. The cells were detached with trypsin-EDTA (Sigma) and transferred to a 6 -well plate at a density of $5 \times 10^{4}$ cells/well. The cells were cultured in serum-free DMEM for $16 \mathrm{~h}$ and then treated with the basal, control, PRL, RA, and PRL plus RA, as described above. The cells were harvested at $\mathrm{d} 1,2,4$, and 8 . Live cells were counted using a hemocytometer after staining cells with 0.04\% trypan blue (Gibco, Grand Island, NY) diluted 1:1 with $1 \times$ PBS.

Total RNA was isolated from MAC-T cells with RNAiso Plus reagent (Takara, Otsu, Japan). The cDNA was prepared with Superscript III (Invitrogen, Carlsbad, CA) according to the manufacturer's instructions. Quantitative PCR was performed on a Bio-Rad CFXConnect optical module (Bio-Rad, Hercules, CA) using SYBR Green (Toyobo, Osaka, Japan) as a marker for DNA amplification. The Q-PCR was performed with $2 \mu \mathrm{L}$ of DNA (input DNA was diluted 1:5) using 40 cycles of 3-step amplification. The primers used for PCR were as follows: GAPDH (NM001034034), GGGTCATCATCTCTGCACCT (forward), GGTCATAAGTCCCTCCACGA (reverse); Bcl-xL (NM001077486), CTCAGAGTAACCGGGAGCTG (forward), CCATTCACAGCAGGGCTATC (reverse); Bax (NM173894), TCTGACGGCAATTTCAACTG (forward), TGGGTGTCCCAAAGTAGGAG (reverse);

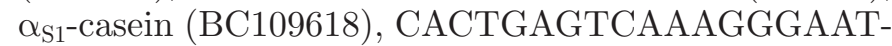
TAAAG (forward), TGATGGCACTTACAGGAGA (reverse); $\quad \alpha_{\mathrm{S}^{-}}$-casein (NM174528.2), CTGGAATTAACTGCTTCTACCT (forward), TACTCTGCGATTTGTCTTATTGA (reverse); $\beta$-casein (NM181008), CCTAACAGCCTCCCACAAAA (forward), AGACTGGAGCAGAGGCAGAG (reverse). Glyceraldehyde-3-phosphate dehydrogenase was used as an internal control because the cycle threshold (Ct) value for GAPDH was not different between the control and treatment samples. The $2^{-\Delta \Delta \mathrm{CT}}$ method (Livak and Schmittgen, 2001) was used to calculate the relative abundance of mRNA.

The general linear models procedure in the SAS software (SAS Institute Inc., Cary, NC) was used to analyze the data from all experiments. Significant differences were determined using the Tukey multiple range test, and $P<0.05$ was considered significant.

All treatment groups exhibited increased proliferation on d 4 of treatment (Figure 1A; $P<0.0001$ ). However, the number of MAC-T cells in the RA treatment was approximately $59 \%$ and $26 \%$ lower than that in the control treatment at d 4 and 8, respectively, indicating that RA inhibited cell proliferation (Figure 1A; $P<$ 0.0001).

As measured by Q-PCR, the ratio of Bcl-xL:Bax in the RA treatment group was decreased compared with that of the control group at $\mathrm{d} 4$ (Figure 1B; $P$ $<0.0001)$. The Bcl-xL:Bax ratio was greater following treatment with PRL alone compared with control, $\mathrm{RA}$, or PRL+RA, and greater with the combination PRL+RA treatment than with RA alone. Thus, one could conclude that all treatments decreased the ratio, but RA, even in combination with PRL, had the greatest effect. The Bcl-xL:Bax ratio was greater in cells grown in the basal medium than in cells grown in the control medium.

Casein gene expression was increased in all treatment groups compared with the control group, particularly the group treated with a combination of RA and PRL (Figure 2). Cells treated with the combination of RA and PRL exhibited an approximately 12.9-fold increase in $\alpha_{S 1}$-casein mRNA (Figure 2A; $P<0.0001$ ) and a 11.9-fold increase in $\alpha_{\mathrm{S}^{2}}$-casein mRNA at $\mathrm{d} 4$ compared with control (Figure 2B; $P<0.0001$ ). Exposure to RA and PRL combined increased the expression of $\beta$-casein mRNA 19.3-fold over that of the control group (Figure $2 \mathrm{C} ; P<0.0001)$.

Lactation is a physiological process characterized by the secretion of large quantities of proteins, carbohydrates, and lipids. For this process to occur, the mammary gland must grow and differentiate through extensive tissue remodeling. The MAC-T cell line, which is derived from the bovine mammary gland, is the most well known in vitro cell line, and it has utility for studying mammary epithelial cell biology and systems. 
A

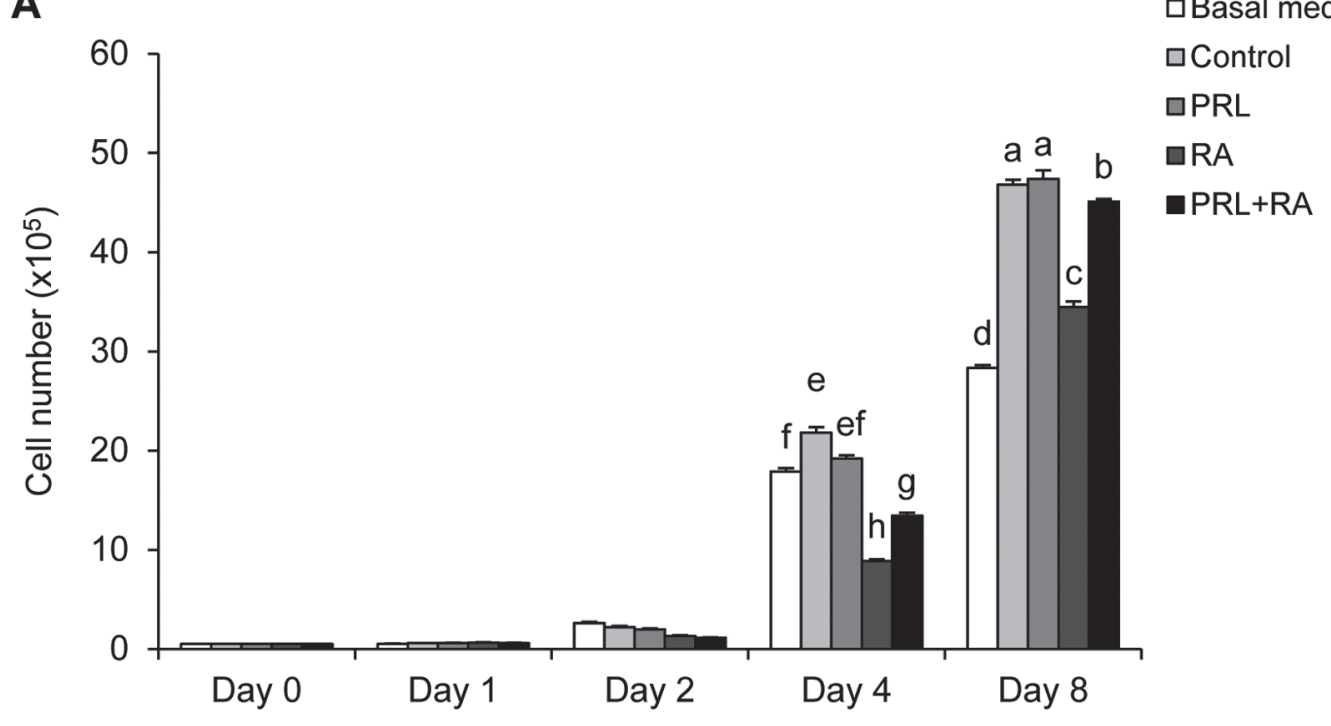

B

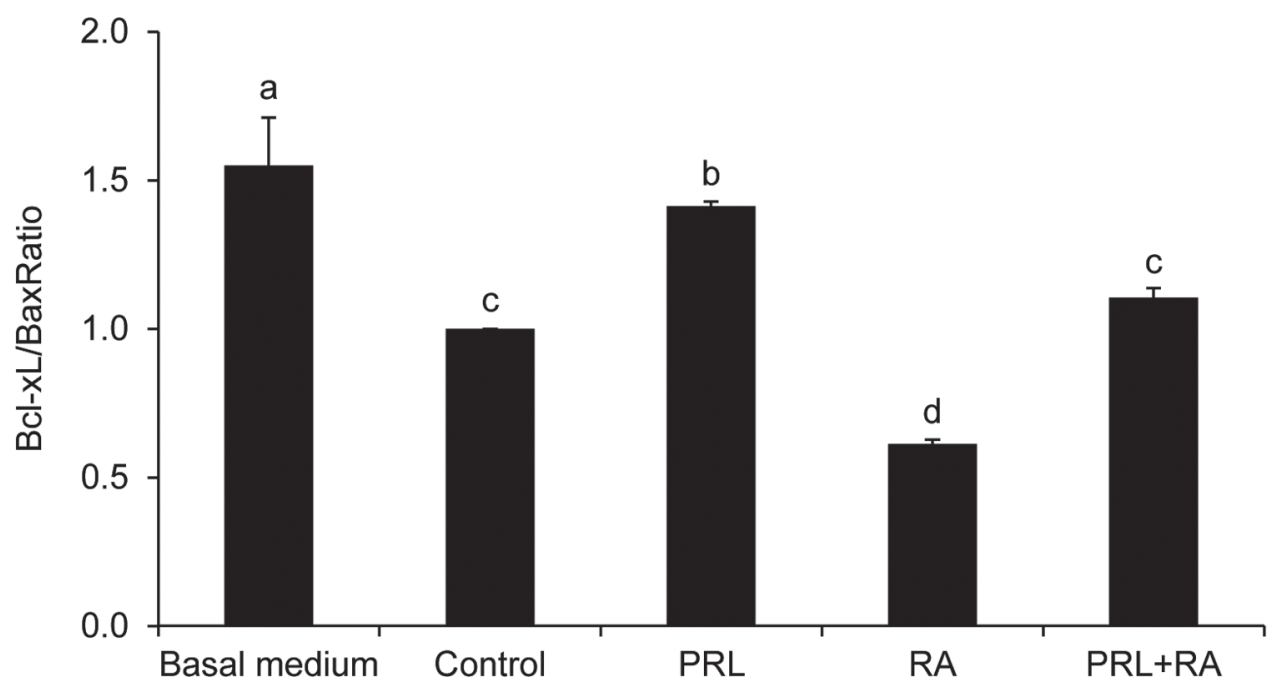

Figure 1. Effects of retinoic acid (RA) on the proliferation, cytotoxicity, and apoptosis of MAC-T cells. The cells were treated with $5 \mu \mathrm{g} /$ $\mathrm{mL}$ prolactin (PRL), $1 \mu \mathrm{M}$ RA, or a combination of PRL and RA in control media up to d 8. Dulbecco's modified Eagle's medium (DMEM) containing $5 \%$ fetal bovine serum was used as the basal treatment. Basal medium was supplemented with $5 \mu \mathrm{g} / \mathrm{mL}$ insulin and $1 \mu \mathrm{g} / \mathrm{mL}$ hydrocortisone for the control treatment. The cell growth curves of the treatment groups were analyzed by cell counting (A), and the cytotoxic and apoptotic effects of various treatments were determined by real-time, reverse transcription-PCR (B). Expression of the apoptosis-related genes $B c l-x L$ and Bax in MAC-T cells was measured at d 4 (B). The MAC-T cells were treated with PRL, RA, or a combination of RA and PRL for $4 \mathrm{~d}$ before total RNA was extracted. Results are expressed as the Bcl-xL:Bax mRNA ratios. Each experiment was repeated 3 times. All values are expressed as mean \pm SEM. ${ }^{\text {a-d, }}$ eh ${ }^{-h}$ etters indicate significant differences between treatments $(P<0.001)$.

In this study, experiments were performed to determine whether supplementation with RA, PRL, or their combination would alter the differentiation of MAC-T cells. Treatment of MAC-T cells with PRL, RA, or a combination induced MAC-T cells to adopt a lactogenic phenotype. Combined treatment with RA and PRL resulted in the most dramatic change in morphology (Supplementary Figure S1; available online at http://www.journalofdairyscience.org/). Although the RA treatment alone induced morphological change of
MAC-T cells, it was much less effective than the combination of RA and PRL. This finding presents strong evidence that RA can induce the morphological changes in MAC-T cells. The data suggest that the effect of RA was distinct from PRL-induced differentiation.

Although treatment with PRL or combined treatment with PRL and RA increased cell proliferation, treatment with RA alone inhibited proliferation. Several studies have demonstrated that RA is involved in the transcriptional regulation of genes that result 
A

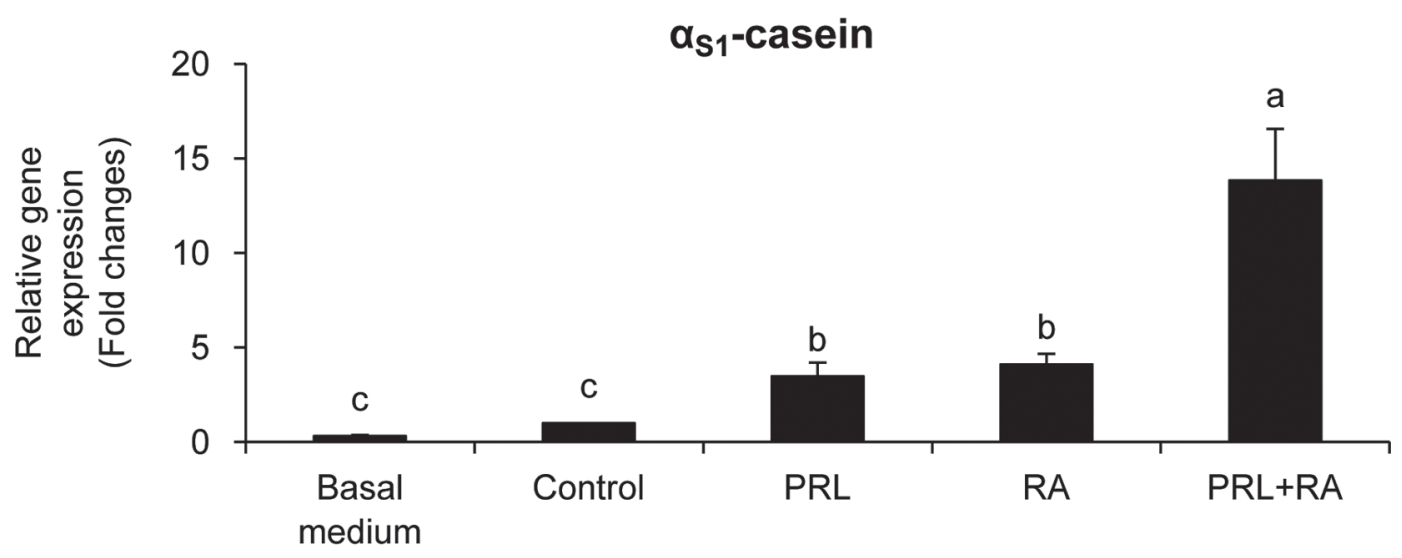

B

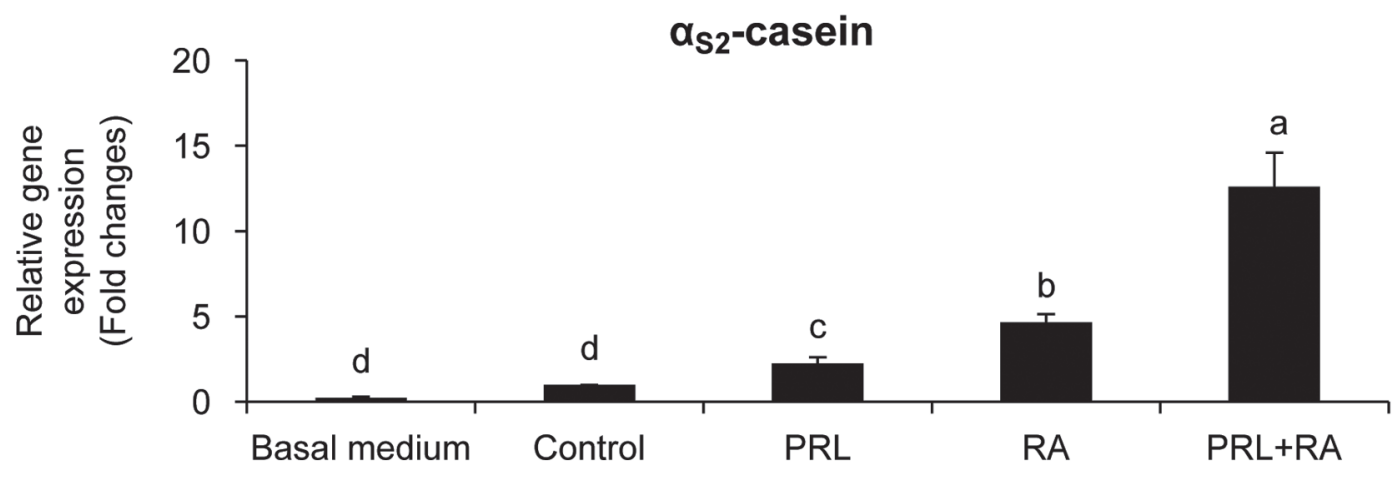

C

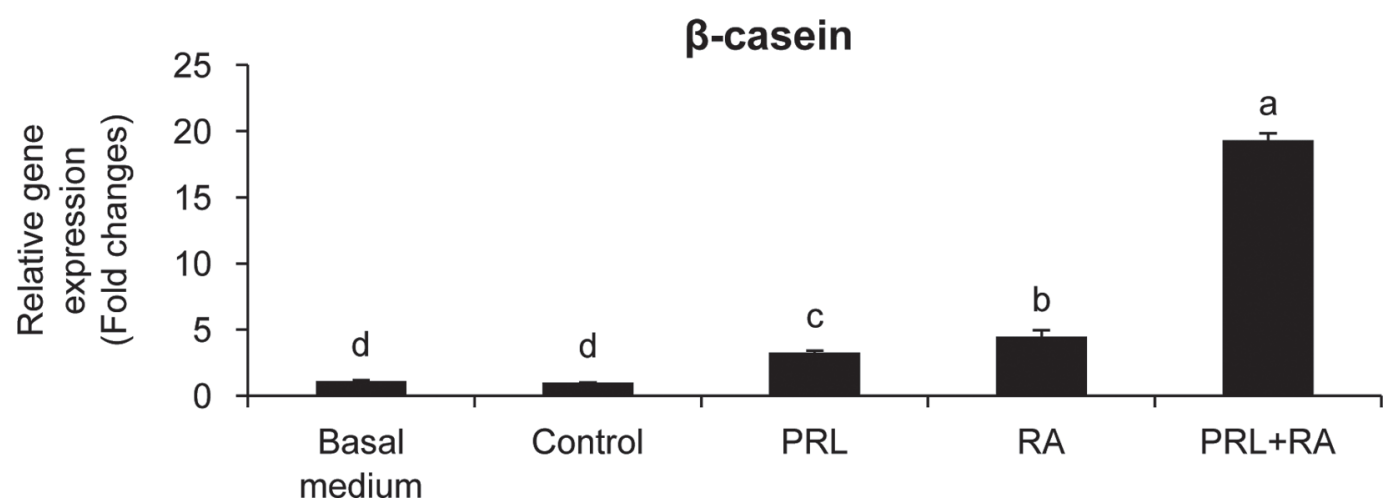

Figure 2. Effect of retinoic acid (RA) on the expression of bovine $\alpha_{\mathrm{S}_{1}}$-casein, $\alpha_{\mathrm{S}^{2}}$-casein, and $\beta$-casein mRNA in MAC-T cells. Dulbecco's modified Eagle's medium (DMEM) containing $5 \%$ fetal bovine serum was used as the basal treatment. Basal medium was supplemented with 5 $\mu \mathrm{g} / \mathrm{mL}$ insulin and $1 \mu \mathrm{g} / \mathrm{mL}$ hydrocortisone for the control treatment. The MAC-T cells were differentiated with $5 \mu \mathrm{g} / \mathrm{mL}$ prolactin (PRL), 1 $\mu M \mathrm{RA}$, or a combination of PRL and RA in control medium for $4 \mathrm{~d}$. Total RNA was isolated, reverse transcribed, and amplified by PCR using gene-specific primers. ${ }^{\mathrm{a}-\mathrm{d}}$ Letters indicate significant differences between treatments $(P<0.001)$.

in growth inhibition, tumor suppression, modulation of differentiation, and cell-to-cell communication (De Luca, 1991; Giguere, 1994; Hossain and Bertram, 1994; Man, 1994; Ruberte, 1994). The depression of proliferation by RA was almost recovered by PRL at $\mathrm{d} 4$ and 8 (Figure 1A).
To identify the effect of RA on apoptosis, we assessed the mRNA expression levels of the apoptosisrelated genes $B c l-x L$ and $B a x$. The Bcl-xL protein is an apoptosis-suppressing factor that heterodimerizes with and inhibits Bax (Golbano et al., 2008). Therefore, the $B c l-x L: B a x$ mRNA ratio is more important than the 
absolute concentration of either $B c l-x L$ mRNA or $B a x$ mRNA (Gross, 2001). The MAC-T cells treated with RA alone exhibited a significant decrease in Bcl-xL:Bax mRNA, indicating that the cells were susceptible to programmed cell death that may have caused the reduction in cell number by RA. In contrast, PRL treatment and the combination of RA and PRL increased the $B c l-x L: B a x$ mRNA ratio compared with control. Apparently, the control medium also increased susceptibility to apoptosis, and this was reversed somewhat by PRL.

Expression of the milk protein casein is an important indicator of the differentiation of MAC-T cells (Ginger and Grigor, 1999). Following treatment with RA or the combined treatment of RA plus PRL, expression of $\alpha_{\mathrm{S1}^{-}}$casein, $\alpha_{\mathrm{S} 2}$-casein, and $\beta$-casein was increased. Prolactin produced a modest increase, whereas the combination of PRL plus RA increased gene expression almost 12-fold. These data support that PRL and RA act synergistically to induce casein expression. Mehta and Moon (1985) reported that addition of PRL or progesterone to medium increased the concentration of cellular retinoic acid binding protein in the mouse mammary gland organ culture. Thus, the synergistic effects of PRL on RA-stimulated casein expression may be mechanistically regulated through PRL induction of RA binding proteins.

Taken together, our findings show that a combination of RA and PRL synergistically increased differentiation and casein mRNA expression. To our knowledge, this is the first report of RA acting as an inducer of differentiation in MAC-T cells. The data suggest that a major function of PRL is to maintain viability of MAC-T cells, whereas RA functions to increase specific gene expression.

\section{ACKNOWLEDGMENTS}

This work was supported by a grant from the Next Generation Biogreen 21 Program (no.PJ008067), RDA, Republic of Korea, and by a research grant from the Chungbuk National University in 2012. We thank S. B. Smith (Texas A\&M University, College Station) for his grammatical revisions to the text.

\section{REFERENCES}

Al-Bataineh, M. M., D. van der Merwe, B. D. Schultz, and R. Gehring. 2010. Tumor necrosis factor alpha increases P-glycoprotein expression in a BME-UV in vitro model of mammary epithelial cells. Biopharm. Drug Dispos. 31:506-515.

Brophy, B., G. Smolenski, T. Wheeler, D. Wells, P. L'Huillier, and G. Laible. 2003. Cloned transgenic cattle produce milk with higher levels of $\beta$-casein and $\kappa$-casein. Nat. Biotechnol. 21:157-162.

De Luca, L. M. 1991. Retinoids and their receptors in differentiation, embryogenesis and neoplasia. FASEB J. 5:2924-2933.

Fukasawa, H., M. Nakagomi, N. Yamagata, H. Katsuki, K. Kawahara, K. Kitaoka, T. Miki, and K. Shudo. 2012. Tamibarotene: A can- didate retinoid drug for Alzheimer's disease. Biol. Pharm. Bull. 35:1206-1212.

Giguere, V. 1994. Retinoic acid receptors and cellular retinoid binding proteins: Complex interplay in retinoid signaling. Endocr. Rev. 15:61-79.

Ginger, M. R., and M. R. Grigor. 1999. Comparative aspects of milk caseins. Comp. Biochem. Physiol. B Biochem. Mol. Biol. 124:133-145.

Golbano, J. M., P. Lóppez-Aparicio, M. N. Recio, and M. A. PérezAlbarsanz. 2008. Finasteride induces apoptosis via Bcl-2, Bcl-xL, Bax and caspase- 3 proteins in $\mathrm{LNCaP}$ human prostate cancer cell line. Int. J. Oncol. 32:919-924.

Gross, A. 2001. Bcl-2 proteins: Regulators of the mitochondrial apoptotic program. IUBMB Life 52:231-236.

He, X. Y., Y. M. Zheng, J. Lan, Y. H. Wu, J. Yan, X. N. He, T. Zhang, Y. L. He, Y. L. Zheng, and Y. Zhang. 2011. Recombinant adenovirus-mediated human telomerase reverse transcriptase gene can stimulate cell proliferation and maintain primitive characteristics in bovine mammary gland epithelial cells. Dev. Growth Differ. $53: 312-322$.

Hossain, M. Z., and J. S. Bertram. 1994. Retinoids suppress proliferation, induce cell spreading, and up-regulate connexin 43 expression only in postconfluent 10T $1 / 2$ cells: Implications for the role of gap junctional communication. Cell Growth Differ. 5:1223-1261.

Huynh, H. T., G. Robitaille, and J. D. Turner. 1991. Establishment of bovine mammary epithelial cells (MAC-T): An in vitro model for bovine lactation. Exp. Cell Res. 197:191-199.

Kumar, S., and G. Duester. 2011. SnapShot: Retinoic acid signaling. Cell 147:1422.

Livak, K. J., and T. D. Schmittgen. 2001. Analysis of relative gene expression data using real time quantitative PCR and the $2^{-\triangle \Delta \mathrm{CT}}$ method. Methods 25:402-408.

Lyons, W. R. 1958. Hormonal synergism in mammary growth. Proc. R. Soc. Lond. B Biol. Sci. 149:303-325.

Man, T. 1994. Solid tumors chemoprevention with retinoids. Leukemia 8:1785-1790.

McCullough, F. S., C. A. Northrop-Clewes, and D. I. Thurnham. 1999. The effect of vitamin A on epithelial integrity. Proc. Nutr. Soc. $58: 289-293$.

Mehta, R. G., and R. C. Moon. 1985. Role of hormones on the induction of retinoic acid binding protein in mouse mammary gland organ culture. Carcinogenesis 6:1103-1107.

Pajot-Augy, E., J. Attal, M. C. Théron, E. Devinoy, M. L. Fontaine, L. M. Houdebine, and R. Salesse. 1997. Recombinant expression and secretion of a natural splicing variant containing the ectodomain of porcine LH receptor in HC11 mammary epithelial cells. Protein Expr. Purif. 10:107-114.

Pecori, G. F., A. G. Ambrogio, M. Andrioli, F. Sanguin, I. Karamouzis, S. M. Corsello, C. Scaroni, E. Arvat, A. Pontercorvi, and F. Cavagnini. 2012. Potential role for retinoic acid in patients with Cushing's disease. J. Clin. Endocrinol. Metab. 97:3577-3583.

Peterson, D. G., E. A. Matitashvili, and D. E. Bauman. 2004. The inhibitory effect of trans-10, cis-12 CLA on lipid synthesis in bovine mammary epithelial cells involves reduced proteolytic activation of the transcription factor SREBP-1. J. Nutr. 134:2523-2527.

Ruberte, E. 1994. Nuclear retinoic acid receptors and regulation of gene expression. Arch. Toxicol. Suppl. 16:105-111.

Sobolewska, A., T. Motyl, and M. Gajewska. 2011. Role and regulation of autophagy in the development of acinar structures formed by bovine BME-UV1 mammary epithelial cells. Eur. J. Cell Biol. 90:854-864.

Zavizion, B., M. van Duffelen, W. Schaeffer, and I. Politis. 1996. Establishment and characterization of a bovine mammary epithelial cell line with unique properties. In Vitro Cell. Dev. Biol. Anim. 32:138-148.

Zhao, C., L. Meng, H. Hu, X. Wang, F. Shi, Y. Wang, Q. Li, and A. Lin. 2010. Spontaneously immortalised bovine mammary epithelial cells exhibit a distinct gene expression pattern from the breast cancer cells. BMC Cell Biol. 11:82. http://dx.doi.org/10.1186/14712121-11-82. 